\title{
Edwards curve points counting method and supersingular Edwards and Montgomery curves (Cryptosystems, Cryptology and Theoretical Computer Science)
}

\author{
RUSLAN SKURATOVSKII, MYKOLA BOHDANENKO, \\ Department of Computer Science, \\ National Technical University of Ukraine, Kiev \\ UKRAINE
}

\begin{abstract}
In this paper, an algebraic affine and projective Edwards curves $[3,9]$ over the finite field $\mathrm{F}_{p^{n}}$ is considered. It is well known, in the theory of Cryptosystems, Cryptology and Theoretical Computer Science, that many modern cryptosystems [11] can be naturally transformed into elliptic curves [5]. In this paper, Edwards algebraic curves over a finite field are studied which are one of the most promising supports of sets of points that are used for fast group operations [1]. In this paper, a new method for counting the order of an Edwards curve over a finite field is presented. This method can be applied in the order of elliptic curves due to the birational equivalence between elliptic curves and Edwards curves. We do not find only a s pecific set of coefficients with corresponding field characteristics for which these curves are supersingular, but we find also a general formula by which one can determine whether a curve $E_{d}\left[\mathrm{~F}_{p}\right]$ is supersingular over this field or not. The embedding degree of the supersingular Edwards curve over $\mathrm{F}_{p^{n}}$ in a finite field is investigated and the field characteristic, where this degree is minimal, is found. A birational isomorphism between the Montgomery curve and the Edwards curve is also constructed. A one-to-one correspondence between the Edwards supersingular curves and Montgomery supersingular curves is presented. The criterion of supersingularity for Edwards curves is found over $F_{p^{n}}$.
\end{abstract}

Keywords: - Cryptosystems, Cryptology, Theoretical Computer Science, Infinite fields, Elliptic curve, Edwards curves, order of group of points of an elliptic curve.

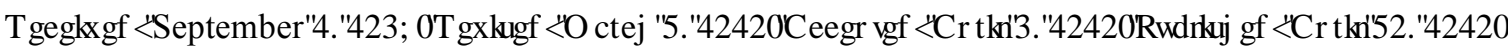

\section{Introduction}

It is well known, in the theory of Cryptosystems, Cryptology and Theoretical Computer Science that many modern cryptosystems [11] can be naturally transformed into elliptic curves [5]. The task of find ing the order of an algebraic curve over a finite field $\mathrm{F}_{p^{n}}$ is now very relevant and is at the center of many $\mathrm{m}$ athematical studies in connection with the use of gr oups of points of curves of genus 1 in cryp tography. In this study, this problem is solved for the Edwards and Montgomery curves.

The criterion of supersingularity of the Edwards curves is found over $\mathrm{F}_{p^{n}}$. We propose also a method for counting the points from Edwards curves and elliptic curves in response to an earlier paper by Schoof [8].

The algebraic affine and projective Edwards curves over a fi nite field is considered. We do not find only a specific set of coefficients with c orresponding field characteristics for which supersingular, but we additionally find a general formula by which one can determine whether a curve $E_{d}\left[\mathrm{~F}_{p}\right]$ is supersingular over this field or not.

\section{Main Result}

The twisted Edwards curve with coefficients $a, d \in F_{p}{ }^{*}, d \neq 1, p \neq 2, a \neq d$, is the curve $E_{a, d}$ : $a x^{2}+y^{2}=1+d x^{2} y^{2}, a, d \in F_{p}^{*}, a d(a-d) \neq 0$,

It should be noted that a twisted Edwards curve is called an Edwards curve when $a=1$.
We denote by $E_{d}$ the Edwards curve with coefficient $d \in F_{p}{ }^{*}$ which is defined as $x^{2}+y^{2}=1+d x^{2} y^{2}$ over $\mathrm{F}_{p}$. The projective curve has form $F(x, y, z)=a x^{2} z^{2}+y^{2} z^{2}=z^{4}+d x^{2} y^{2}$.

The special points are the infinitely distant points $(1,0,0)$ and $(0,1,0)$ and $t$ herefore we fi nd its singularities at infinity in the corresponding affine components $A^{1}:=a z^{2}+y^{2} z^{2}=z^{4}+d y^{2}$ and $A^{2}: a x^{2} z^{2}+z^{2}=z^{4}+d x^{2}$. These are simple singularities.

We describe the structure of the local ring at the point $p_{1}$ whose elements are quotients of functions with the form $F(x, y, z)=\frac{f(x, y, z)}{g(x, y, z)}$, where the denominator cannot take the value of 0 at the singular point $p_{1}$. In particular, we note that a local ring which has two singularities consists of functions with the denominators are not divisible by $(x-1)(y-1)$.

We denote by $\delta_{\mathrm{p}}=\operatorname{dim}^{\overline{\mathrm{o}}_{\mathrm{p}}} / \mathrm{O}_{\mathrm{p}}$, where $\mathrm{O}_{\mathrm{p}}$ denotes the local ring at the si ngular point $p$ which is generated by the relations of regular functions $\mathrm{O}_{\mathrm{p}}=\left\{\frac{f}{g}:(g,(x-1)(y-1))=1\right\}$ and $\overline{\mathrm{O}}_{\mathrm{p}}$ denotes the whole closure of the local ring at the singular point $p$. 
We find that $\delta_{p}=\operatorname{dim}^{\bar{O}_{p}} /_{\mathrm{O}_{p}}=1$ is the dimension of the factor as a ve ctor space. Because the basis of e xtension $\overline{\mathrm{O}}_{p} / \mathrm{O}_{p}$ consists of just one el ement at each distinct point, we obtain that $\delta_{p}=1$. We calculate then the genus of the curve according to Fulton [4].

$$
\rho^{*}(C)=\rho_{\alpha}(C)-\sum_{p \in E} \delta_{p}=\frac{(n-1)(n-2)}{2}-\sum_{p \in E} \delta_{p}=3-2=1,
$$

where $\rho_{\alpha}(C)$ denotes the arithmetic genus of the curve $C$ with parameter $n=\operatorname{deg}(C)=4$. It should be noted that the supersingular points were discovered in [10]. We recall the curve has a genus of 1 and as such it is known to be isomorphic to a flat c ubic curve, however, the curve is importantly not elliptic because of its si ngularity in the projective part. Both the Edwards curve and the twisted Edwards curve are isomorphic to some affine part of the elliptic curve. The Edwards curve after normalization is precisely a curve in the Weierstrass normal form, which was proposed by Montgomery [1] and will be denoted by $E_{M}$.

Koblitz [4,5] proves that one can d etect if a cu rve is supersingular using the search for the curve when that curve has the same number of points as its torsion curve. Also an elliptic curve $E$ over $F_{q}$ is called supersingular if for every finite extension $F_{q^{r}}$ there are no points in the group $E\left(F_{q^{r}}\right)$ of order $p[17]$. It is kno wn [1] that the transition from an Ed wards curve to the related torsion curve is determined by the reflection $(\bar{x}, \bar{y}) \mapsto(x, y)=\left(\bar{x}, \frac{1}{\bar{y}}\right)$. We now recall an important result from Vinogradov [13] which will act as criterion for supersingularity.

Lemma 2.1. Let $k \in \square$ and $p \in \mathrm{P}$. Then

$\sum_{k=1}^{p-1} k^{n} \equiv \begin{cases}0(\bmod p), & n \nmid(p-1), \\ -1(\bmod p), & n \mid(p-1),\end{cases}$

where $n \mid(p-1)$ denotes that $n$ is divisible by $p-1$.

The order of a curve is precisely the number of its affine points with a neutral element, where the group operation is well defined. It is known that the order of

$x^{2}+y^{2}=1+d x^{2} y^{2}$ coincides with the order of the curve $x^{2}+y^{2}=1+d^{-1} x^{2} y^{2}$ over $F_{p}$. We will now strengthen an existing result given in [10]. We denote the number of points with a neutral element of an a ffine Edwards curve over the finite field $\mathrm{F}_{p}$ by $N_{d[p]}$ and the number of points on the projective curve over the same field by $\bar{N}_{d[p]}$.

Theorem 2.1. If $p \equiv 3(\bmod 4)$ is prime and the following condition of supersingularity

$$
\sum_{j=0}^{\frac{p-1}{2}}\left(C_{\frac{p-1}{2}}^{j}\right)^{2} d^{j} \equiv 0(\bmod p)
$$

is true then the orders of the curves $x^{2}+y^{2}=1+d x^{2} y^{2}$ and $x^{2}+y^{2}=1+d^{-1} x^{2} y^{2}$ over $F_{p}$ are equal to

$N_{d[p]}=p+1$, when $\left(\frac{d}{p}\right)=-1$, and $N_{d[p]}=p-3$, when $\left(\frac{d}{p}\right)=1$.

Proof. Consider the curve $E_{d}$ :

$$
x^{2}+y^{2}=1+d x^{2} y^{2} .
$$

Transform it into the form $y^{2}\left(1-d x^{2} y^{2}\right)=1-x^{2}$, then we express $y^{2}$ by applying a rational transformation which lead us to the curve $y^{2}=\frac{1-x^{2}}{1-d x^{2} y^{2}}$.

For our analysis we transform it into the curve

$$
y^{2}=\left(x^{2}-1\right)\left(d x^{2}-1\right) .
$$

We denote the number of points from an af fine Edwards curve over the finite field $\mathrm{F}_{p}$ by $M_{d[p]}$. This curve (3) has $M_{d[p]}=N_{d[p]}+\left(\frac{d}{p}\right)+1$ points, which is precisely $\left(\frac{d}{p}\right)+1$ greater than the number of points of curve $E_{d}$. Note that $\left(\frac{d}{p}\right)$ denotes the Legendre Symbol. Let $a_{0}, a_{1}, \ldots, a_{2 p-2}$ be the coefficients of the polynomial $a_{0}+a_{1} x+\ldots+a_{2 p-2} x^{2 p-2}$, which was obtained from $\left(x^{2}-1\right)^{\frac{p-1}{2}}\left(d x^{2}-1\right)^{\frac{p-1}{2}}$ after opening the brackets. Thus, summing over all $x$ yields $M_{d[p]}=\sum_{x=0}^{p-1} 1+\left(\left(x^{2}-1\right)\left(d x^{2}-1\right)\right)^{\frac{p-1}{2}}=p+\sum_{x=0}^{p-1}\left(x^{2}-1\right)^{\frac{p-1}{2}}$. $\cdot\left(d x^{2}-1\right)^{\frac{p-1}{2}} \equiv \sum_{x=0}^{p-1}\left(x^{2}-1\right)^{\frac{p-1}{2}}\left(d x^{2}-1\right)^{\frac{p-1}{2}}(\bmod p)$.

By opening the brackets in $\left(x^{2}-1\right)^{\frac{p-1}{2}}\left(d x^{2}-1\right)^{\frac{p-1}{2}}$, we have $a_{2 p-2}=(-1)^{\frac{p-1}{2}} \cdot d^{\frac{p-1}{2}} \equiv\left(\frac{d}{p}\right)(\bmod p)$. So, using Lemma 2.1 we have

$$
M_{d[p]} \equiv-\left(\frac{d}{p}\right)-a_{p-1}(\bmod p) .
$$

We need to prove that $M_{d[p]} \equiv 1(\bmod p)$ if $p \equiv 3(\bmod 8)$ and $M_{d[p]} \equiv-1(\bmod p)$. We have to show therefore that $M_{d[p]} \equiv-\left(\frac{d}{p}\right)-a_{p-1}(\bmod p) \quad$ for $\quad p \equiv 3(\bmod 4) \quad$ if $\sum_{j=0}^{\frac{p-1}{2}}\left(C_{\frac{p-1}{2}}^{j}\right)^{2} d^{j} \equiv 0(\bmod p)$. If we can pr ove that $a_{p-1} \equiv 0(\bmod p)$, then it will fo llow from (3). Let us determine $a_{p-1}$ according to Newton's binomial formula: $a_{p-1}$ is equal to the coefficient at $x^{p-1}$ in the polynomial, which is obtained as a product $\left(x^{2}-1\right)^{\frac{p-1}{2}}\left(d x^{2}-1\right)^{\frac{p-1}{2}}$. So, 
$a_{p-1}=(-1)^{\frac{p-1}{2}} \sum_{j=0}^{\frac{p-1}{2}} d^{j}\left(C_{\frac{p-1}{2}}^{j}\right)^{2}$. Actually, the following equality holds:

$$
\begin{aligned}
& \sum_{j=0}^{\frac{p-1}{2}} d^{j}\left(C_{\frac{p-1}{2}}^{\frac{p-1}{2}-j}\right)(-1)^{\frac{p-1}{2}-\left(\frac{p-1}{2}-j\right)} \cdot d^{j}\left(C_{\frac{p-1}{2}}^{j}\right)^{2}(-1)^{\frac{p-1}{2}-j}= \\
& =(-1)^{\frac{p-1}{2}} \sum_{j=0}^{\frac{p-1}{2}} d^{j} C_{\frac{p-1}{2}}^{\frac{p-1}{2}-j} \cdot C_{\frac{p-1}{2}}^{j}=(-1)^{\frac{p-1}{2}} \sum_{j=0}^{\frac{p-1}{2}} d^{j}\left(C_{\frac{p-1}{2}}^{j}\right)^{2} .
\end{aligned}
$$

Since $a_{p-1}=-\sum_{j=0}^{\frac{p-1}{2}}\left(C_{\frac{p-1}{2}}^{j}\right)^{2} d^{j}$, then exact number of affine points on non supersingular curve is the following $M_{d[p]} \equiv-a_{2 p-2}-a_{p-1} \equiv-\left(\frac{d}{p}\right)+\sum_{j=0}^{\frac{p-1}{2}}\left(C_{\frac{p-1}{2}}^{j}\right)^{2} d^{j}(\bmod p)$.

According to th $\mathrm{e}$ condition of this theorem $a_{p-1}=0$, therefore $M_{d[p]} \equiv-a_{2 p-2}(\bmod p)$. Consequently, in the case when $p \equiv 3(\bmod 4)$, where $p$ is prime and $\sum_{j=0}^{\frac{p-1}{2}}\left(C_{\frac{p-1}{2}}^{j}\right)^{2} d^{j} \equiv 0(\bmod p)$, the curve $E_{d}$ has $N_{d[p]}=p-\left(\frac{d}{p}\right)-\left(\left(\frac{d}{p}\right)+1\right)=p-1-2\left(\frac{d}{p}\right)$ affine points and a group of points of the curve completed by singular points has $p+1$ points.

The exact number of the points has upper bound $2 p+1$ because for every $x \neq 0$ corresponds two valuations of $y$, but for $x=0$ we have only one solution $y=0$. Taking into account that $x \in F_{p}$ we have exactly $p$ values of $x$. Also there are 4 pairs $( \pm 1,0)$ and $(0, \pm 1)$ which are points of $E_{d}$ thus $N_{d[p]}>1$. Thus $N_{d[p]}=p+1$. This completes the proof.

Corollary: The orders of the curves $x^{2}+y^{2}=1+d x^{2} y^{2}$ and $x^{2}+y^{2}=1+d^{-1} x^{2} y^{2} \quad$ over $\quad F_{p}$ are equal to $N_{d[p]}=p+1=\bar{N}_{d[p]}, \quad$ when $\left(\frac{d}{p}\right)=-1$,

and $\quad N_{d[p]}=p-3=\bar{N}_{d[p]}-4, \quad$ when $\quad\left(\frac{d}{p}\right)=1 \quad$ iff $p \equiv 3(\bmod 4)$ is prime and $\sum_{j=0}^{\frac{p-1}{2}}\left(C_{\frac{p-1}{2}}^{j}\right)^{2} d^{j} \equiv 0(\bmod p)$.

Since all transformations in proof of Theorem 2.1. were equivalent transitions then we o btain the proof of equivalence of conditions.

Theorem 2.2. If $\mathrm{t}$ he coefficient $d=2$ or $d=2^{-1}$ and $p \equiv 3(\bmod 4)$ then $\sum_{j=0}^{\frac{p-1}{2}} d^{j}\left(C_{\frac{p-1}{d}}^{j}\right)^{2} \equiv 0(\bmod p)$ and $\bar{N}_{d[p]}=p+1$.

Proof. When $p \equiv 3(\bmod 4)$, we shall show that $\sum_{j=0}^{\frac{p-1}{2}} d^{j}\left(C_{\frac{p-1}{d}}^{j}\right)^{2} \equiv 0(\bmod p)$. We multiply each binomial coefficient in this sum by $\left(\frac{p-1}{2}\right)$ ! to obtain after som e algebraic manipulation $\left(\frac{p-1}{2}\right) ! C_{\frac{p-1}{2}}^{j}=\frac{\left(\frac{p-1}{2}\right)\left(\frac{p-1}{2}-1\right) \cdots\left(\frac{p-1}{2}-j+1\right)\left(\frac{p-1}{2}\right) !}{1 \cdot 2 \cdots j}=$
$=\left(\frac{p-1}{2}\right)\left(\frac{p-1}{2}-1\right) \cdots\left(\frac{p-1}{2}-j+1\right)\left[\left(\frac{p-1}{2}\right)\left(\frac{p-1}{2}-1\right) \cdots(j+1)\right]$.

After that, by applying the congruence $\left(\frac{p-1}{2}-k\right)^{2} \equiv\left(\frac{p-1}{2}+1+k\right)^{2}(\bmod p)$ with $0 \leq k \leq \frac{p-1}{2}$ to the multipliers in previous parentheses, we obtain $\left[\left(\frac{p-1}{2}\right)\left(\frac{p-1}{2}-1\right) \cdots(j+1)\right]$.

It yields

$$
\begin{aligned}
& \left(\frac{p-1}{2}\right)\left(\frac{p-1}{2}-1\right) \cdots\left(\frac{p-1}{2}-j+1\right) . \\
& {\left[\left(\frac{p-1}{2}+1\right) \cdots\left(\frac{p-1}{2}+\frac{p-1}{2}-j\right)\right](-1)^{\frac{p-1}{2}-j} .}
\end{aligned}
$$

Thus, as a result of squaring, we have:

$$
\begin{aligned}
& \left(\left(\frac{p-1}{2}\right) ! C_{\frac{p-1}{2}}^{j}\right)^{2} \equiv\left(\frac{p-1}{2}-j+1\right)^{2}\left(\frac{p-1}{2}-j+2\right)^{2} . \\
& \cdots(p-j-1)^{2}(\bmod p) .
\end{aligned}
$$

It remains to prove that $\sum_{j=0}^{\frac{p-1}{2}}\left(C_{\frac{p-1}{2}}^{j}\right)^{2} 2^{j} \equiv 0(\bmod p)$ if $p \equiv 3(\bmod 4)$.

Consider the auxiliary polynom

ial $P(t)=\left(\frac{p-1}{2} !\right)^{2} \sum_{j=0}^{\frac{p-1}{2}}\left(C_{\frac{p-1}{2}}^{j}\right)^{2} t^{j}$. We are going to show that $P(2)=0$ and therefore $a_{p-1} \equiv 0(\bmod p)$. Using (6) it can be shown that $a_{p-1}=P(t)=\left(\frac{p-1}{2} !\right)^{2} \sum_{j=0}^{\frac{p-1}{2}}\left(C_{\frac{p-1}{2}}^{j}\right)^{2} t^{j} \equiv \sum_{j=0}^{\frac{p-1}{2}}(k+1)^{2}$.

$\cdot(k+2)^{2} \ldots\left(\frac{p-1}{2}+k\right)^{2} t^{k}(\bmod p)$

over $F_{p}$. We replace $d$ by $t$ in (1) such that we can research a more generalized problem. It should be noted that

$P(t)=\partial^{\frac{p-1}{2}}\left(\partial^{\frac{p-1}{2}}\left(Q(t) t^{\frac{p-1}{2}}\right) t^{\frac{p-1}{2}}\right) \quad$ over $\quad F_{p}, \quad$ where $Q(t)=t^{p-1}+\ldots+t+1 \quad$ and $\partial^{\frac{p-1}{2}}$ denotes the $\frac{p-1}{2}$-th derivative by $t$, where $t$ is new variable but not a coordinate of curve. Observe that 
$Q(t)=\frac{t^{p}-1}{t-1} \equiv \frac{(t-1)^{p}}{t-1} \equiv(t-1)^{p-1}(\bmod p)$ and therefore the equality $P(t)=\left(\left((t-1)^{p-1} t^{\frac{p-1}{2}}\right)^{\left(\frac{p-1}{2}\right)} t^{\frac{p-1}{2}}\right)^{\left(\frac{p-1}{2}\right)}$ holds over $\mathrm{F}_{p}$. In order to simplify the notation, we let $\theta=t-1$ and $R(\theta)=P(\theta+1)$. For the case $t=2$ we have $\theta=1$. Performing this substitution leads the polynomial $P(t)$ of 2 to the polynomial $R(t-1)$ of 1 . Taking into account the linear nature of the substitution $\theta=t-1$, it can be seen that that derivation by $\theta$ and $t$ coincide. Derivation leads us to the transformation of polynomial $R(\theta)$ to form where it has the necessary coefficient $a_{\mathrm{p}-1}$. Then $R(\theta)=P(\theta+1)=\partial^{\frac{p-1}{2}}\left(\partial^{\frac{p-1}{2}}\left(\theta^{p-1}(\theta+1)^{\frac{p-1}{2}}\right)(\theta+1)^{\frac{p-1}{2}}\right)=$ $=\partial^{\frac{p-1}{2}}\left(\frac{(p-1) !}{((p-1) / 2) !} \theta^{\frac{p-1}{2}}(\theta+1)^{\frac{p-1}{2}}\right)$.

In order to prove that $a_{p-1} \equiv 0(\bmod p)$, it is now sufficient to show that $R(\theta)=0$ if $\theta=1$ over $F_{p}$. We obtain

$$
R(1)=\frac{(p-1) !}{\left(\frac{p-1}{2}\right) !} \sum_{j=0}^{\frac{p-1}{2}} C_{\frac{p-1}{2}}^{j}(j+1) \cdots\left(j+\frac{p-1}{2}\right) .
$$

We will manipulate now the expression $\left(\frac{p-1}{2}-j+1\right)\left(\frac{p-1}{2}-j+2\right) \cdots\left(\frac{p-1}{2}-j+\frac{p-1}{2}\right)$.

In order to illustrate the simplification we now consider the scenario when $p=11$ and hence $\frac{p-1}{2}=5$.

The expression gets the

form

$(5-j+1)(5-j+2) \cdots(5-j+5)=(6-j)(7-j) \cdots(10-j) \equiv$

$\equiv((-5-j)(-4-j) \cdots(-1-j)) \equiv$

$\equiv(-1)^{5}((j+1)(j+2) \cdots(j+5))(\bmod 11)$.

Therefore, for a prime $p$, we can rewrite the expression as $\left(\frac{p-1}{2}-j+1\right)\left(\frac{p-1}{2}-j p-2\right) \cdots\left(\frac{p-1}{2}-j+\frac{p-1}{2}\right) p_{2}=1 \quad p$ $\equiv(-1)^{\frac{p-1}{2}}(j+1) \quad(j+-) \equiv-1(j+1) \quad(j+\longrightarrow)(\bmod )$.

As a result, the symmetrical terms in (7) can be reduced yielding $a_{p-1} \equiv 0(\bmod p)$. It should be noted that $(-1)^{\frac{p-1}{2}}=-1 \quad$ since $\quad p=M k+3$ and $\frac{p-1}{2}=2 k+1$. Consequently, we ha ve $P(2)=R(1)=0$ and hence $a_{p-1} \equiv 0(\bmod p) \quad$ as required. Thus, $\sum_{j=0}^{\frac{p-1}{2}}\left(C_{\frac{p-1}{2}}^{j}\right)^{2} \equiv 0(\bmod p)$, completing the proof of the of the theorem.

Corollary 2.2. The c urve $E_{d}$ is supersingular iff $E_{d^{-1}}$ is supersingular.
Proof. Let us recall the proved fact in Theorem 2.1 that $N_{d[p]} \equiv-a_{2 p-2}-a_{p-1} \equiv-\left(\frac{d}{p}\right)+\sum_{j=0}^{\frac{p-1}{2}}\left(C_{\frac{p-1}{2}}^{j}\right)^{2} d^{j}(\bmod p)$.

Since $\left(C_{\frac{p-1}{2}}^{j}\right)^{2} d^{j} \equiv 0(\bmod p)$ by condition, and the congruence $\left(\frac{d}{p}\right) \equiv\left(\frac{d^{-1}}{p}\right)$ holds, then $N_{d[p]} \equiv N_{d^{-1}[p]}$.

Corollary 2.3. If $p \equiv 3(\bmod 4)$, is prime then there exists some $T$ such that $T \equiv \sum_{j=0}^{\frac{p-1}{2}}\left(C_{\frac{p-1}{2}}^{j}\right)^{2} d^{j} \leq 2 \sqrt{q} \quad$ and $N_{d[p]}=p-1-2\left(\frac{d}{p}\right)+T$.

Proof. Due to equality (5) a nd the bounds (8) as well as according to generalized Hasse-Weil theorem $\left|N_{d[p]}-(p+1)-2\left(\frac{d}{p}\right)\right| \leq 2 g \sqrt{p}$, where $g$ is genus of curve, we obtain exact number $N_{d[p]}$ as we showed, $g=1$.

From Theorem 2.1 as well as from Corollary 2.2 we get, that $\sum_{j=0}^{\frac{p-1}{2}}\left(C_{\frac{p-1}{2}}^{j}\right)^{2} d^{j} \equiv-N_{d[p]}-(p+1)-2\left(\frac{d}{p}\right)$ so there exists $T \in \square$, such that $T<2 \sqrt{p}$ and $N_{d[p]}=p-1-2\left(\frac{d}{p}\right)+T$.

Example 2.1. If $p=13, d=2$ gives $N_{2[13]}=8$ and $p=13$, $d^{-1}=7$ gives that the $\mathrm{n}$ umber of points of $E_{7}$ is $N_{7[13]}=20$, which is in contradiction to that suggested by Bessalov and Thsigankova Moreover, if $p \equiv 7(\bmod 8)$, then the order of torsion subgroup of curve is $N_{2}=N_{2^{-1}}=p-3$, which is clearly different to $p+1$

For instance $p=31$, then $N_{2[31]}=N_{2^{-1}[31]}=28=31-3$, which is clearly not equal to $p+1$. If $p=7, d=2^{-1} \equiv(4 \bmod 7)$ then the curve $E_{2^{-1}}$ has four points, namely $(0,1) ;(0,6) ;(1,0) ;(6,0)$, and the in case $p=7$ with $d=2(\bmod 7)$, the curve $E_{2^{-1}}$ also has fo ur points: $(0,1) ;(0,6) ;(1,0) ;(6,0)$, demonstrating the order in this scenario is $p-3$.

The following theorem shows that the total number of affine points upon the Edwards curves $E_{d}$ and $E_{d^{-1}}$ are equal under certain assumptions. This the orem provides us additionally with a formula for enumerating the number of affine points upon the birationally isomorphic Montgomery curve $N_{M}$. 
Theorem 2.3. Let $d$ satisfy the condition of supersingularity (1). If $n \equiv 1(\bmod 2)$ and $p$ is prime, then $\bar{N}_{d\left[p^{n}\right]}=p^{n}+1$ and the order of curve is equal to

$$
N_{d\left[p^{n}\right]}=p^{n}-1-2\left(\frac{d}{p}\right)
$$

If $n \equiv 0(\bmod 2)$ and $p$ is prime, then the order of curve $N_{d\left[p^{n}\right]}=p^{n}-3-2(-p)^{\frac{n}{2}}$, and the order of projective curve is equal to $\bar{N}_{d\left[p^{n}\right]}=p^{n}+1-2(-p)^{\frac{n}{2}}$.

If $n \equiv 0(\bmod 2)$ and $p$ is prime, then the order of projective curve is equal to

$\bar{N}_{d\left[p^{n}\right]}=p^{n}+1-2(-p)^{n / 2}$, and The order of curve is equal to $N_{d\left[p^{n}\right]}=p^{n}+3-2(-p)^{n / 2}$.

Proof. We consider the extension of the base field $F_{p}$ to $F_{p^{n}}$ in order to determine the number of the points on the curve $x^{2}+y^{2}=1+d x^{2} y^{2}$. Let $P(x)$ denotes a polynomial with degree $m>2$ whose coefficients are from $\mathrm{F}_{p}$.To make the proof, we take $\mathrm{i}$ nto account that it is known that the nu mber of solutions to $y^{2}=P(x)$ over $\mathrm{F}_{p^{n}} \quad$ will have $\mathrm{t}$ he form $p^{n}+1-\omega_{1}^{n}-\ldots-\omega_{m-1}^{n}$ where $\omega_{1}, \ldots, \omega_{m-1} \in \square,\left|\omega_{i}\right|=p^{\frac{1}{2}}$.

In case of our supersingular curve, if $n \equiv 1(\bmod 2)$ the number of points on projective curve over $\mathrm{F}_{p^{n}}$ is determined by the expression $p^{n}+1-\omega_{1}^{n}-\omega_{2}^{n}$, where $\omega_{i}^{n} \in \square$ and $\omega_{1}=-\omega_{2},\left|\omega_{i}\right|=\sqrt{p}$ that's why $\omega_{1}=i \sqrt{p}$, $\omega_{2}=-i \sqrt{p}$ with $i \in\{1,2\}$. In the general case, it is known that $\left|\omega_{i}\right|=p^{\frac{1}{2}}$. The order of the projective curve is therefore $p^{n}+1$.

If $p \equiv 7(\bmod 8)$, then it is known from a result of Skuratovskii [10] that $E_{d}$ has in its projective closure of the curve singular points which are not affine and therefore

$$
N_{d[p]}=p^{n}-3 .
$$

If $p \equiv 3(\bmod 8)$, then there are no singular points, hence

$$
\bar{N}_{d[p]}=N_{d[p]}=p^{n}+1 \text {. }
$$

Consequently the number of points on the Edwards curve depends on $\left(\frac{d}{p}\right)$ and is equal to $N_{d[p]}=p^{n}-3$ if $p \equiv 7(\bmod 8)$ and $N_{d[p]}=p^{n}+1$ if $p \equiv 3(\bmod 8)$ where $n \equiv 1(\bmod 2)$. We note that this is because the transformation of (3) in $E_{d}$ depends upon the denominator $\left(d x^{2}-1\right)$.

If $n \equiv 1(\bmod 2)$ then, with respect to the sum of the root of the characteristic equation for the Frobenius endomorphism $\omega_{1}^{n}+\omega_{2}^{n}$, which in this case have the same signs, we obtain that the number of points in the group of points of the curve is $p^{n}+1-\omega_{1}^{n}-\omega_{2}^{n}$ [19]. In $\mathrm{m}$ ore details $\omega_{1}, \omega_{2}$ are eigenvalues of Frobenius operator $F$ endomorphism on etale cohomology over the finite field $\mathrm{F}_{p^{n}}$, where $F$ acts of $H^{i}(X)$. The number of points, in gene ral case, are determined by Lifshitz formula:

$$
\# X\left(\mathrm{~F}_{p^{n}}\right)=\sum(-1)^{i} \operatorname{tr}\left(\mathrm{F}^{n} \mid H^{i}(X)\right)
$$

where $\# X\left(\mathrm{~F}_{p^{n}}\right)$ is a number of points in the $\mathrm{m}$ anifold $X$ over $\mathrm{F}_{p^{n}}, F^{n}$ is composition of the Frobenius operator. In our case, $E_{d}$ is considered as the manifold $X$ over $\mathrm{F}_{p^{n}}$.

For $n \equiv 0(\bmod 2)$ we always have, that every $d \in F_{p}$ is a quadratic residue in $F_{p^{n}}$. Consequently, because of $\left(\frac{d}{p}\right)=1$ four singular points appear on the curve. Thus, the number of affine points is less by 4, i.e. $N_{d\left[p^{n}\right]}=p^{n}-1-2\left(\frac{d}{p}\right)-2(-p)^{\frac{n}{2}}=p^{n}-3-2(-p)^{\frac{n}{2}}$.

Lemma 2.2. There exists birational isomorphism between $E_{d}$ and $E_{M}$, which is determ ined by correspondent mappings $x=\frac{1+u}{1-u}$ and $y=\frac{2 u}{v}$.

Proof. To verify this statement in supersingular case we suppose that the curve $x^{2}+y^{2}=1+d x^{2} y^{2}$ contains $p-1-2\left(\frac{d}{p}\right)$ points $(x, y)$, with coordinates over prime field $\mathrm{F}_{p}$. Consider the tra nsformation of the curve $x^{2}+y^{2}=1+d x^{2} y^{2} \quad$ into the following form $y^{2}\left(d x^{2}-1\right)=x^{2}-1$. Make the substitutions $x=\frac{1+u}{1-u}$ and $y=\frac{2 u}{v}$. We will call the special points of this transformations the point in which these transformations or inverse transformations are not determined. As a result the equation of curve the equation of the curve takes the form $\frac{4 u^{2}}{v^{2}} \cdot \frac{(d-1) u^{2}+2(d+1) u+(d-1)}{(1-u)^{2}}=\frac{4 u}{(1-u)^{2}}$. Multiply the equation of the curve by $\frac{v^{2}(1-u)^{2}}{4 u}$. As a result of the reduction, we obtai $\mathrm{n}$ the equation $v^{2}=(d-1) u^{3}+2(d+1) u^{2}+(d-1) u$. We analyze what new solutions appeared in $\mathrm{t}$ he resulting equation in com paring 
$Q(t)=\frac{t^{p}-1}{t-1} \equiv \frac{(t-1)^{p}}{t-1} \equiv(t-1)^{p-1}(\bmod p)$ and therefore the equality $P(t)=\left(\left((t-1)^{p-1} t^{\frac{p-1}{2}}\right)^{\left(\frac{p-1}{2}\right)} t^{\frac{p-1}{2}}\right)^{\left(\frac{p-1}{2}\right)}$ holds over $\mathrm{F}_{p}$. In order to simplify the notation, we let $\theta=t-1$ and $R(\theta)=P(\theta+1)$. For the case $t=2$ we have $\theta=1$. Performing this substitution leads the polynomial $P(t)$ of 2 to the polynomial $R(t-1)$ of 1 . Taking into account the linear nature of the substitution $\theta=t-1$, it can be seen that that derivation by $\theta$ and $t$ coincide. Derivation leads us to the transformation of polynomial $R(\theta)$ to form where it has the necessary coefficient $a_{\mathrm{p}-1}$. Then $R(\theta)=P(\theta+1)=\partial^{\frac{p-1}{2}}\left(\partial^{\frac{p-1}{2}}\left(\theta^{p-1}(\theta+1)^{\frac{p-1}{2}}\right)(\theta+1)^{\frac{p-1}{2}}\right)=$ $=\partial^{\frac{p-1}{2}}\left(\frac{(p-1) !}{((p-1) / 2) !} \theta^{\frac{p-1}{2}}(\theta+1)^{\frac{p-1}{2}}\right)$.

In order to prove that $a_{p-1} \equiv 0(\bmod p)$, it is now sufficient to show that $R(\theta)=0$ if $\theta=1$ over $F_{p}$. We obtain

$$
R(1)=\frac{(p-1) !}{\left(\frac{p-1}{2}\right) !} \sum_{j=0}^{\frac{p-1}{2}} C_{\frac{p-1}{2}}^{j}(j+1) \cdots\left(j+\frac{p-1}{2}\right) .
$$

We will manipulate now the expression $\left(\frac{p-1}{2}-j+1\right)\left(\frac{p-1}{2}-j+2\right) \cdots\left(\frac{p-1}{2}-j+\frac{p-1}{2}\right)$.

In order to illustrate the simplification we now consider the scenario when $p=11$ and hence $\frac{p-1}{2}=5$.

The expression gets the

form

$(5-j+1)(5-j+2) \cdots(5-j+5)=(6-j)(7-j) \cdots(10-j) \equiv$

$\equiv((-5-j)(-4-j) \cdots(-1-j)) \equiv$

$\equiv(-1)^{5}((j+1)(j+2) \cdots(j+5))(\bmod 11)$.

Therefore, for a prime $p$, we can rewrite the expression as $\left(\frac{p-1}{2}-j+1\right)\left(\frac{p-1}{2}-j+2\right) \cdots\left(\frac{p-1}{2}-j+\frac{p-1}{2}\right) \equiv$ $\equiv(-1)^{\frac{p-1}{2}}(j+1) \cdots\left(j+\frac{p-1}{2}\right) \equiv-1(j+1) \cdots\left(j+\frac{p-1}{2}\right)(\bmod p)$.

As a result, the symmetrical terms in (7) can be reduced yielding $a_{p-1} \equiv 0(\bmod p)$. It should be noted that $(-1)^{\frac{p-1}{2}}=-1 \quad$ since $\quad p=M k+3$ and $\frac{p-1}{2}=2 k+1$. Consequently, we ha ve $P(2)=R(1)=0$ and hence $a_{p-1} \equiv 0(\bmod p) \quad$ as required. Thus, $\sum_{j=0}^{\frac{p-1}{2}}\left(C_{\frac{p-1}{2}}^{j}\right)^{2} \equiv 0(\bmod p)$, completing the proof of the of the theorem.

Corollary 2.2. The c urve $E_{d}$ is supersingular iff $E_{d^{-1}}$ is supersingular.
Proof. Let us recall the proved fact in Theorem 2.1 that $N_{d[p]} \equiv-a_{2 p-2}-a_{p-1} \equiv-\left(\frac{d}{p}\right)+\sum_{j=0}^{\frac{p-1}{2}}\left(C_{\frac{p-1}{2}}^{j}\right)^{2} d^{j}(\bmod p)$.

Since $\left(C_{\frac{p-1}{2}}^{j}\right)^{2} d^{j} \equiv 0(\bmod p)$ by condition, and the congruence $\left(\frac{d}{p}\right) \equiv\left(\frac{d^{-1}}{p}\right)$ holds, then $N_{d[p]} \equiv N_{d^{-1}[p]}$.

Corollary 2.3. If $p \equiv 3(\bmod 4)$, is prime then there exists some $T$ such that $T \equiv \sum_{j=0}^{\frac{p-1}{2}}\left(C_{\frac{p-1}{2}}^{j}\right)^{2} d^{j} \leq 2 \sqrt{q} \quad$ and $N_{d[p]}=p-1-2\left(\frac{d}{p}\right)+T$.

Proof. Due to equality (5) a nd the bounds (8) as well as according to generalized Hasse-Weil theorem $\left|N_{d[p]}-(p+1)-2\left(\frac{d}{p}\right)\right| \leq 2 g \sqrt{p}$, where $g$ is genus of curve, we obtain exact number $N_{d[p]}$ as we showed, $g=1$.

From Theorem 2.1 as well as from Corollary 2.2 we get, that $\sum_{j=0}^{\frac{p-1}{2}}\left(C_{\frac{p-1}{2}}^{j}\right)^{2} d^{j} \equiv-N_{d[p]}-(p+1)-2\left(\frac{d}{p}\right)$ so there exists $T \in \square$, such that $T<2 \sqrt{p}$ and $N_{d[p]}=p-1-2\left(\frac{d}{p}\right)+T$.

Example 2.1. If $p=13, d=2$ gives $N_{2[13]}=8$ and $p=13$, $d^{-1}=7$ gives that the $\mathrm{n}$ umber of points of $E_{7}$ is $N_{7[13]}=20$, which is in contradiction to that suggested by Bessalov and

Thsigankova

Moreover, if $p \equiv 7(\bmod 8)$, then the order of torsion subgroup of curve is $N_{2}=N_{2^{-1}}=p-3$, which is clearly different to $p+1$

For instance $p=31$, then $N_{2[31]}=N_{2^{-1}[31]}=28=31-3$, which is clearly not equal to $p+1$. If $p=7, d=2^{-1} \equiv(4 \bmod 7)$ then the curve $E_{2^{-1}}$ has four points, namely $(0,1) ;(0,6) ;(1,0) ;(6,0)$, and the in case $p=7$ with $d=2(\bmod 7)$, the curve $E_{2^{-1}}$ also has fo ur points: $(0,1) ;(0,6) ;(1,0) ;(6,0)$, demonstrating the order in this scenario is $p-3$.

The following theorem shows that the total number of affine points upon the Edwards curves $E_{d}$ and $E_{d^{-1}}$ are equal under certain assumptions. This the orem provides us additionally with a formula for enumerating the number of affine points upon the birationally isomorphic Montgomery curve $N_{M}$. 
algebraic extension of degree $n$, we will consider $p^{n}-\omega_{1}^{n}-\omega_{2}^{n}=p^{n} \quad$ if $n \equiv 1(\bmod 2)$. Therefore, for $n \equiv 1(\bmod 2)$, the order of the Montgomery curve is precisely given by $N_{M\left[p^{n}\right]}=p^{n}+1$. Here's one infinitely remote point as a neutral element of the group of points of the curve.

Considering now an elliptic cu rve, we $h$ ave $\omega_{1}=\bar{\omega}_{2}$ by [5], which leads to $\omega_{1}+\omega_{2}=0$. For $n=1$, it is clear that $N_{M}=p$. When $n$ is odd, we have $\omega_{1}^{n}+\omega_{2}^{n}=0$ and therefore $N_{M, n}=p^{n}+1$. Because $n$ is even by initial assumption, we sh all show that $N_{M\left[p^{n}\right]}=p^{n}+1-2(-p)^{\frac{n}{2}}$ holds as required.

Note that for $n=2$ we can express the number as $\bar{N}_{d\left[p^{2}\right]}=p^{2}+1+2 p=(p+1)^{2}$ with respect to Lagran ge theorem have to be divisible on $\bar{N}_{d[p]}$. Because a group of $E_{d}\left(F_{p^{2}}\right)$ over square extension of $F_{p}$ contains the group $E_{d}\left(\mathrm{~F}_{p}\right)$ as a proper subgroup. In fact, according to Theorem 1 the order $E_{d}\left(\mathrm{~F}_{p}\right)$ is $p+1$ therefore divisibility of order $E_{d}\left(\mathrm{~F}_{p^{2}}\right)$ holds because in our case $p=7$ thus $\bar{N}_{E_{d}}=8^{2}$ and $p+1=8=N_{d[7]}[16]$.

The following two examples exemplify Corollary 2.4.

Example 2.3. If $p \equiv 3(\bmod 8)$ and $n=2 k$ then we have when $d=2, n=2, p=3$ that the number of affine points equals to $N_{2[3]}=p^{n}-3-2(-p)^{\frac{n}{2}}=3^{2}-3-2 \cdot(-3)=12$, and the number of projective points is equal to $\bar{N}_{2[3]}=p^{n}+1-2(-p)^{\frac{n}{2}}=3^{2}+1-2 \cdot(-3)=16$.

Example 2.4. If $p \equiv 7(\bmod 8)$ and $n=2 k$ then we have when $d=2, n=2, p=7$ that the number of affine points equals to $N_{2[7]}=p^{n}-3-2(-p)^{\frac{n}{2}}=7^{2}-3-2 \cdot(-7)=60$, and the number of projective points is equal to $\bar{N}_{2[7]}=p^{n}+1-2(-p)^{\frac{n}{2}}=7^{2}+1-2 \cdot(-7)=64$.

The group of points of the supersingular curve $E_{d}$ contains $p-1-2\left(\frac{d}{p}\right)$ affine points and the affine singular points whose number is $2\left(\frac{d}{p}\right)+2$.

The singular points were discovered in [10] and hence if the curve is free of singular points then the group order is $p+1$.

Example 2.5.1 The number of curve points over finite field when $d=2$ and $p=31$ is equal to $N_{2[31]}=N_{2^{-1}[31]}=p-3=28$.
Theorem 2 2.4. The order of Edwards curve over $F_{p}$ is congruent to

$$
\begin{aligned}
& \bar{N}_{d[p]} \equiv\left(p-1-2\left(\frac{d}{p}\right)+(-1)^{\frac{p+1}{2}} \sum_{j=0}^{\frac{p-1}{2}}\left(C_{\frac{p-1}{2}}^{j}\right)^{2} d^{j}\right) \equiv \\
& \equiv\left((-1)^{\frac{p+1}{2}} \sum_{j=0}^{\frac{p-1}{2}}\left(C_{\frac{p-1}{2}}^{j}\right)^{2} d^{j}-1-2\left(\frac{d}{p}\right)\right)(\bmod p) .
\end{aligned}
$$

The true value of $\bar{N}_{d[p]}$ lies in [4;2p] and is even.

Proof. This result follows from the number of solutions of the equation $y^{2}=\left(x^{2}-1\right)\left(d x^{2}-1\right)$ over $F_{p}$ which equals to

$$
\begin{aligned}
& \left.\left.\sum_{x=0}^{p-1}\left(\frac{\left(x^{2}-1\right)\left(d x^{2}-1\right)}{p}\right)+1\right) \equiv \sum_{x=0}^{p-1}\left(\frac{\left(x^{2}-1\right)\left(d x^{2}-1\right)}{p}\right)\right)+p \equiv \\
& \equiv\left(\sum_{j=0}^{\frac{p-1}{2}}\left(x^{2}-1\right)^{\frac{p-1}{2}}\left(d x^{2}-1\right)^{\frac{p-1}{2}}\right)(\bmod p) \equiv \\
& \equiv\left((-1)^{\frac{p+1}{2}} \sum_{j=0}^{\frac{p-1}{2}}\left(C_{\frac{p-1}{2}}^{j}\right)^{2} d^{j}-\left(\frac{d}{p}\right)\right)(\bmod p) .
\end{aligned}
$$

The quantity of solutions for $x^{2}+y^{2}=1+d x^{2} y^{2}$ differs from the quantity of $y^{2}=\left(d x^{2}-1\right)\left(x^{2}-1\right)$ by $\left(\frac{d}{p}\right)+1$ due to new solutions in the from $(\sqrt{d}, 0),(-\sqrt{d}, 0)$. So this quantity is such

$$
\begin{aligned}
& \left.\sum_{x=0}^{p-1}\left(\frac{\left(x^{2}-1\right)\left(d x^{2}-1\right)}{p}\right)+1\right)-\left(\left(\frac{d}{p}\right)+1\right) \equiv \\
& \left.\sum_{x=0}^{p-1}\left(\frac{\left(x^{2}-1\right)\left(d x^{2}-1\right)}{p}\right)\right)+p-\left(\left(\frac{d}{p}\right)-1\right) \equiv \\
& \equiv\left(\sum_{j=0}^{\frac{p-1}{2}}\left(x^{2}-1\right)^{\frac{p-1}{2}}\left(d x^{2}-1\right)^{\frac{p-1}{2}}-\left(\frac{d}{p}\right)+1\right)(\bmod p) \equiv \\
& \equiv(-1)^{\frac{p+1}{2}} \sum_{j=0}^{\frac{p-1}{2}}\left(C_{\frac{p-1}{2}}^{j}\right)^{2} d^{j}-\left(2\left(\frac{d}{p}\right)+1\right)(\bmod p) .
\end{aligned}
$$

According to Lemma 1 th e last su m $\left(\sum_{j=0}^{\frac{p-1}{2}}\left(x^{2}-1\right)^{\frac{p-1}{2}}\left(d x^{2}-1\right)^{\frac{p-1}{2}}\right)(\bmod p)$ is congruent to $-a_{p-1}-a_{2 p-2}(\bmod p)$, where $a_{i}$ are the coefficients from presentation

$$
\left(x^{2}-1\right)^{\frac{p-1}{2}}\left(d x^{2}-1\right)^{\frac{p-1}{2}}=a_{0}+a_{1} x+\ldots+a_{2 p-2} x^{2 p-2} .
$$


Last presentation was obtained due $\mathrm{t} o$ transformation

$$
\begin{aligned}
& \left(x^{2}-1\right)^{\frac{p-1}{2}}\left(d x^{2}-1\right)^{\frac{p-1}{2}}=\left(\sum_{x=0}^{p-1} C_{\frac{p-1}{2}}^{k} x^{2 k}(-1)^{\frac{p-1}{2}-k}\right) \\
& \left(\sum_{x=0}^{p-1} C_{\frac{p-1}{2}}^{j} d^{j} x^{2 j}(-1)^{\frac{p-1}{2}-j}\right) .
\end{aligned}
$$

Therefore $a_{2 p-2}$ is equal to $d^{\frac{p-1}{2}} \equiv\left(\frac{d}{p}\right)(\bmod p)$ and $a_{p-1}=\sum_{j=0}^{\frac{p-1}{2}}\left(C_{\frac{p-1}{2}}^{j}\right)^{2} d^{j}(-1)^{\frac{p-1}{2}}$.

According to Newton's binomial formula $a_{p-1}$ equal to $t$ he coefficient at $x^{p-1}$ in the product of two brackets and when substituting it $d$ instead of 2 is such

$$
(-1)^{\frac{p-1}{2}} \sum_{j=0}^{\frac{p-1}{2}} d^{j}\left(C_{\frac{p-1}{2}}^{j}\right)^{2}
$$

that is, it has the form of the polynomial with inverse order of coefficients.

Indeed, we have equality

$$
\begin{aligned}
& \sum_{j=0}^{\frac{p-1}{2}} d^{j}\left(C_{\frac{p-1}{2}}^{\frac{p-1}{2}-j}\right)(-1)^{\frac{p-1}{2}-\left(\frac{p-1}{2}-j\right)} \cdot\left(C_{\frac{p-1}{2}}^{j}\right)^{2}(-1)^{\frac{p-1}{2}-j}= \\
& =(-1)^{\frac{p-1}{2}} \sum_{j=0}^{\frac{p-1}{2}} d^{j} C_{\frac{p-1}{2}}^{\frac{p-1}{2}-j} \cdot C_{\frac{p-1}{2}}^{j}=(-1)^{\frac{p-1}{2}} \sum_{j=0}^{\frac{p-1}{2}} d^{j}\left(C_{\frac{p-1}{2}}^{j}\right)^{2} . \\
& \text { In form of a su } \mathrm{m} \text { it is th } \mathrm{e} \text { following } \\
& \sum_{j=0}^{\frac{p-1}{2}} 2^{j}\left(C_{\frac{p-1}{2}}^{\frac{p-1}{2}-j}\right)(-1)^{\frac{p-1}{2}-\left(\frac{p-1}{2}-j\right)} \cdot 2^{j}\left(C_{\frac{p-1}{2}}^{j}\right)^{2}(-1)^{\frac{p-1}{2}-j}= \\
& =(-1)^{\frac{p-1}{2}} \sum_{j=0}^{\frac{p-1}{2}} 2^{j} C_{\frac{p-1}{2}}^{\frac{p-1}{2}-j} \cdot C_{\frac{p-1}{2}}^{j}=(-1)^{\frac{p-1}{2}} \sum_{j=0}^{\frac{p-1}{2}} 2^{j}\left(C_{\frac{p-1}{2}}^{j}\right)^{2} . \\
& \text { over } \quad F_{p} \quad \text { equals } \\
& p-1-2\left(\frac{d}{p}\right)+\left(1+\left(\frac{d}{p}\right)\right)=p-\left(\frac{d}{p}\right) \text { and differs from the } \\
& \text { quantity of solutions of } x^{2}+y^{2}=1+d x^{2} y^{2} \text { by }\left(\frac{d}{p}\right)+1 \text { due } \\
& N_{E_{d}}=\left(p-\left(\frac{d}{p}\right)-\left(\left(\frac{d}{p}\right)+1\right)-(-1)^{\frac{p-1}{2}} \sum_{j=0}^{\frac{p-1}{2}}\left(C_{\frac{p-1}{2}}^{\frac{p-1}{2}-j} C_{\frac{p-1}{2}}^{j}\right)^{2} d^{j}\right) \equiv \\
& \equiv\left(p-1-(-1)^{\frac{p-1}{2}} \sum_{j=0}^{\frac{p-1}{2}}\left(C_{\frac{p-1}{2}}^{j}\right)^{2} d^{j}-2\left(\frac{d}{p}\right)\right) \equiv \\
& \equiv\left((-1)^{\frac{p+1}{2}} \sum_{j=0}^{\frac{p-1}{2}}\left(C_{\frac{p-1}{2}}^{j}\right)^{2} d^{j}-1-2\left(\frac{d}{p}\right)\right)(\bmod p) \text {. }
\end{aligned}
$$

The exact order is not less than 4 because cofactor of this curve is 4 . To determine the order is uniquely enough to take into account that $p$ and $2 p$ have different parity. Taking into account that the order is even we chose a term $p$ or $2 p$, for the sum which define the order.

Theorem 2.5.3 If $\left(\frac{d}{p}\right)=1$, then the orders of the curves $E_{d}$ and $E_{d^{-1}}$, satisfies to the following relation $\left|E_{d}\right|=\left|E_{d^{-1}}\right|$.

$$
\text { If }\left(\frac{d}{p}\right)=-1 \text {, then } E_{d} \text { and } E_{d^{-1}} \text { are pair of twisted }
$$

curves i.e. orders of curves $E_{d}$ and $E_{d^{-1}}$ satisfies to the following relation of duality

$$
\left|E_{d}\right|+\left|E_{d^{-1}}\right|=2 p+2
$$

Let the curve be $d$ efined by $x^{2}+y^{2}=1+d x^{2} y^{2}(\operatorname{modp})$, then we can express $y^{2}$ in such way:

$$
y^{2} \equiv \frac{x^{2}-1}{d x^{2}-1}(\bmod p
$$

For $x^{2}+y^{2}=1+d^{-1} x^{2} y^{2}(\bmod p)$ we could obtain that

$$
\begin{aligned}
& y^{2} \equiv \frac{x^{2}-1}{d^{-1} x^{2}-1}(\bmod p) \\
& \text { If }\left(\frac{d}{p}\right)=1 \text {, then for the fixed } x_{0} \text { a quantity of } y
\end{aligned}
$$

over $\mathrm{F}_{p}$ can be calculated by the formula $\left(\frac{\frac{x^{2}-1}{d^{-1} x^{2}-1}}{p}\right)+1$ for $x$ such that $d^{-1} x^{2}+1 \equiv 0(\bmod p)$. For so lution $\left(x_{0}, y_{0}\right)$ to (10), we have the equality $y_{0}^{2} \equiv \frac{x_{0}^{2}-1}{d x_{0}^{2}-1}(\bmod p)$ and we express

$y_{0}^{2} \equiv \frac{1-\frac{1}{x_{0}^{2}}}{1-\frac{1}{d x_{0}^{2}}} d^{-1} \equiv \frac{\left(\frac{1}{x_{0}}\right)^{2}-1}{\frac{1}{d}\left(\frac{1}{x_{0}}\right)^{2}-1} d^{-1} \equiv \frac{\left(\frac{1}{x_{0}}\right)^{2}-1}{d^{-1}\left(\frac{1}{x_{0}}\right)^{2}-1} d^{-1}$.

Observe that

$$
\begin{aligned}
& y^{2}=\frac{x^{2}-1}{d^{-1} x^{2}-1}=\frac{1-x^{2}}{1-d^{-1} x^{2}}=\frac{\left(\frac{1}{x^{2}}-1\right)}{\left(\left(\frac{d}{x^{2}}\right)-1\right)} d . \\
& \text { Thus, if }\left(x_{0}, y_{0}\right) \text { is solution of }(2), \text { then }\left(\frac{1}{x_{0}}, \frac{y_{0}}{\sqrt{d}}\right)
\end{aligned}
$$
is a solution to (10) because last transformations determines 
that $\frac{\mathrm{y}_{0}^{2}}{d} \equiv \frac{d^{-1}\left(\frac{1}{x_{0}}\right)^{2}-1}{\left(\frac{1}{x_{0}}\right)^{2}-1} \operatorname{modp}$. Therefore last transformations $\left(x_{0}, y_{0}\right) \rightarrow\left(\frac{1}{x_{0}}, \frac{y_{0}}{\sqrt{d}}\right)=(x, y)$ determines isomorphism and bijection.

In case $\left(\frac{d}{p}\right)=-1$, then every $x \in \mathrm{F}_{p}$ is such that $d x^{2}-1 \neq 0$ and $d^{-1} x^{2}-1 \neq 0$. If $x_{0} \neq 0$, then $x_{0}$ generate 2 solutions of (2) iff $x_{0}^{-1}$ gives 0 solutions of (10) because of (11) yields the following relation

$$
\left(\frac{\frac{x^{2}-1}{d^{-1} x^{2}-1}}{p}\right)=\left(\frac{\frac{x^{-2}-1}{d x^{-2}-1}}{p}\right)\left(\frac{d}{p}\right)=-\left(\frac{\frac{x^{-2}-1}{d x^{-2}-1}}{p}\right) .
$$

Analogous reasons give us that $x_{0}$ give exactly one solution of (2) iff $x_{0}^{-1}$ gives 1 solutions of (10). Consider the set $x \in\{1,2, \ldots, p-1\}$ we obtain that the total amount of solutions of form $\left(x_{0}^{-1}, y_{0}\right)$ that represent point of (2) and pairs of form $\left(x_{0}, y_{0}\right)$ that represent point of curve (10) is $2 p-2$. Also we have two solutions of $(2)$ of form $(0,1)$ and $(0,-1)$ and two solutions of $(10)$ that has form $(0,1)$ and $(0,-1)$. The proof is fully completed.

Example 2.6. 4 The number of points of $E_{d}$ over $F_{p}$ for $p=13$ and $d=2$ is given by $N_{2[13]}=8$. In the case when $p=13$ and $d^{-1}=7$ we have that the number of points of $E_{7}$ is $N_{7[13]}=20$. Therefore, we have that the sum of orders for these curve is equal to $28=2 \cdot 13+2$ which confirms our theorem. The set of points over $F_{13}$ when $d=2$ are precisely

$\{(0,1) ;(0,12) ;(1,0) ;(4,4) ;(4,9) ;(9,4) ;(9,9) ;(12,0)\}, \quad$ while for $d=7$, we have the set $\{(0,1) ;(0,12) ;(1,0) ;(2,4) ;(2,9) ;(4,2) ;(4,11) ;(5,6) ;(5,7)$; $(6,5) ;(6,8) ;(7,5) ;(7,8) ;(8,6) ;(8,7) ;(9,2) ;(9,11) ;(11,4) ;(11,9)$; $(12,0)\}$.

Example 2.7. 5 If $p=7$ and $d=2^{-1} \equiv 4(\bmod 7)$, then we have $\left(\frac{d}{p}\right)=1$ and the curve $E_{2^{-1}}$ has four points which are $(0,1) ;(0,6) ;(1,0) ;(6,0)$. and the in case $p=7$ for $d=2(\bmod 7)$, the curve $E_{2^{-1}}$ also has four points which are $(0,1) ;(0,6) ;(1,0) ;(6,0)$.

Definition 2.1. 6 We call the embedding degree a minimal power $k$ of a finite field extension such that the group of points of the curve can be embedded in the multiplicative group of $\mathrm{F}_{p^{k}}$.
Let us obtain conditions of embedding [14] for the group of supersingular curves $E_{d}\left[\mathrm{~F}_{p}\right]$ of order $p$ in the multiplicative group of field $\mathrm{F}_{p^{k}}$ whose embedding degree is $k=12$ [14]. We n ow utilise the Zsigmondy theorem which implies that a suitable characteristic of field $\mathrm{F}_{p}$ is an arbitrary prime $p$ which do not divide 12 and satisfies the condition $q \mid \mathrm{P}_{12}(p)$, where $\mathrm{P}_{12}(x)$ is the cyclotomic polynomial. This $p$ will satisfy the $\mathrm{n}$ ecessary conditions $\left(x^{n}-1\right) \| p$ for an arbitrary $n=1, \ldots, 11$.

Proposition 2.2.7 The degree of embedding for the group of a supersingular curve $E_{d}$ is equal to 2 .

Proof. The order of the group of a supersingular curve $E_{d}$ is equal to $p^{k}+1$. It should be observed that $p^{k}+1$ divides $p^{2 k}-1$, but $p^{k}+1$ does not divide expressions of the form $p^{2 l}-1$ with $l<k$. This division does not work for smaller values of $l$ due to the decomposition of the expression $p^{2 k}-1=\left(p^{k}-1\right)\left(p^{k}+1\right)$. Therefore, we can use the definition to conclude that the degree of immersion must be 2, confirming the proposition.

Consider $\mathrm{E}_{2}$ over $\mathrm{F}_{p^{2}}$, for i nstance we a ssume $p=3$. We define $\mathrm{F}_{9}$ as $\mathrm{F}_{3}(\alpha)$, where $\alpha$ is a $\mathrm{r}$ oot of $x^{2}+1=0$ over $F_{9}$. Therefore elements of $F_{9}$ have form: $a+b \alpha$, where $a, b \in \mathrm{F}_{3}$. So we a ssume that $x \in\{ \pm(\alpha+1), \pm(\alpha-1), \pm \alpha\}$ and check its belonging to $\mathrm{E}_{2}$. For instance if $x= \pm(\alpha+1)$ then $x^{2}=\alpha^{2}+2 \alpha+1=2 \alpha=-\alpha$. Also in this case $y^{2}=\frac{2 \alpha-1}{\alpha-1}=\frac{(2 \alpha-1)(\alpha+1)}{(\alpha-1)(\alpha+1)}=\frac{(2 \alpha-1)(\alpha+1)}{(\alpha-1)(\alpha+1)}=\frac{\alpha}{-2}=\alpha$.

Therefore the correspondent second coordinate is $y= \pm(\alpha-1)$. The similar computations lead us to full the following list of curves points.

Points of Edwards curve over square extension.

The total amount is 12 affine points that confirms Corollary 2.4. and $\mathrm{Th}$ eorem 2.3. b ecause of $p^{n}-3-2(-p)^{\frac{n}{2}}=3^{2}-3-2(-3)=12$.

\section{Conclusion}

A new method for the order curve counting for Edwards and elliptic curves has been presented. The criterion for supersingularity of these curves was also obtained.

Acknowledgement: We thanks to Alexandr Rybak for interesting disscussion.

\section{References}

[1] Daniel J. B ernstein, Peter Birkner, Marc Joye, Tanja Lange, and Christiane Peters. Twisted edwards curves. In Serge Vaudenay, editor, Progress in Cryp tology -- 
AFRICACRYPT 2008, pages 389--405, Berlin, Heidelberg, 2008. Springer Berlin Heidelberg.

[2] A.V Bessalov and O.V. Thsigankova. Vzaimosvyaz' semeystva tochek bol'shikh poryadkov krivoy edvardsa nad prostym polem (in russian). Information Security, 17(1):73--80, 2015.

[3] Harold Edwards. A normal form for elliptic cur ves. Bulletin of the American mathematical society, 44(3):393--422, 2007.

[4] William Fulton. Al gebraic curves. An Introduction to Algebraic Geometry. Add ison-Wesley, 3 edition, 2008.L. Gu, L. Wang, K. Ota, M. Dong, Z. Cao and Y. Yang, New public key cryptosystems based on nonabelian factorization problems, Secur. Commun. Netw. Vol 6, 2013, no. 7, pp. 912-922.

[5] Neal Koblitz. Ellip tic curve cryptosystems. Mathematics of computation, 48(177):203--209, 1987.

[6] Rudolf Lidl and Harald Niederreiter. In troduction to Finite Fields and their Applications. Cambridge university press, 1994.

[7] Peter L Montgomery. Speeding the pollard and elliptic curve methods of factorization. Mathe matics of computation, 48(177):243--264, 1987.

[8] René Schoof. Counting points on elliptic curves over finite fields. Journal de théorie des nombres de Bordeaux, 7(1):219--254, 1995.

[9] Ruslan Viacheslavovich Skuratovskii. T he order of projective edwards curve over and embedding degree of this curve in finite field. In Cait 2018, Proceedings of Conferences, pages 75 -- 80, 2018.

[10] Ruslan Viacheslavovich

Skuratovskii. Supersingularity of elliptic curves over (in ukrainian). Research in Mathematics and Mechanics, 31(1):17--26, 2018.

[11] Ruslan Viacheslavovich Skuratovskii. Employment of minimal generating sets and structure of sylow 2subgroups alternating groups in block ciphers. In
Advances in Computer Communication and Computational Sciences, pages 351--364. Springer, 2019.

[12] Serge Aleksandrovich Stepanov. Arifmetika algebraicheskikh krivykh (in Russian). Nauka, Glav. red. fiziko-matematichesko lit-ry, 1991.

[13] Ivan Matveevich Vinogradov. Elements of number theory. Courier Dover Publications, 2016.

[14] Paulo S. L. M. Barreto a nd Michael Naehrig. Pairingfriendly elliptic curves of prime order. In Bart Preneel and Stafford Tavares, editors, Selected Areas in Cryptography, pages 319--331, Berlin, Heidelberg, 2006. Springer Berlin Heidelberg.

[15] N.M. Glazunov, Skobelev S.P. Manifolds over the rings. IAMM National Academy of Sciences of Ukraine, Donetsk, 2011.323 p.

[16] P.D Varbanec, P Zarzycki. Divisors of the Gaussian integers in an arithmetic progression. Journal of Number Theory. Volume 33, Issue 2, October 1989, Pages 152-169

[17] Silverman, Joseph, H.; The Arith metic of Elliptic Curves, Graduate Texts in Mathematics, 106, SpringerVerlag, 1986.

[18] R. V. Skuratovskii, Aled Williams (2019) "A solution of the inverse problem to doubling of twisted Edwards curve point over finite fiel d", Processing, transmission and security of information - 2019 vol. 2, Wydawnictwo Naukowe Akademii TechnicznoHumanistycznej w Bielsku-Bialej

[19] Deligne, Pierre. La conjecture de Weil, Publications Mathematiques de l'IHES. 1974. Vol. 43. pp. 273307.IEEE

[20] R. V. Skuratovskii, Employment of Minimal Generating Sets and St ructure of Sylow 2-Subgroups Alternating Groups in Block Ciphers. Springer, Advances in Computer Communication and Computational Sciences, 2019, pp. 351-364. 